\title{
Low Blood Pressure, Low Serum Cholesterol and Anemia Predict Early Necessity of Ventricular Assist Device Implantation in Patients With Advanced Heart Failure at the Time of Referral From Non-Ventricular Assist Device Institutes
}

Takeo Fujino, MD, PhD; Koichiro Kinugawa, MD, PhD; Masaru Hatano, MD;

Teruhiko Imamura, MD, PhD; Hironori Muraoka, MD; Shun Minatsuki, MD;

Toshiro Inaba, MD, PhD; Hisataka Maki, MD, PhD; Osamu Kinoshita, MD, PhD;

Kan Nawata, MD, PhD; Atsushi Yao, MD, PhD; Minoru Ono, MD, PhD; Issei Komuro, MD, PhD

\begin{abstract}
Background: The timing of ventricular assist device (VAD) implantation is always a matter of debate, especially when a patient is referred from a non-VAD institute. We focused on objective noninvasive parameters at the time of admission to a VAD implant center and analyzed the factors predicting the necessity of early VAD.
\end{abstract}

\begin{abstract}
Methods and Results: We retrospectively analyzed advanced heart failure (HF) patients referred since January 2011 , including patients less than 65 years old. They all had a history of hospitalization for HF management in non-VAD institutes within 1 month before referral. We excluded patients transferred with mechanical circulatory support. We enrolled 46 patients (40 males, 39.8 \pm 13.4 years old). Among them, 26 patients had a VAD implanted or died within 120 days. By multivariable logistic analysis using admission parameters, systolic blood pressure (BP) $<93 \mathrm{mmHg}$ [odds ratio (OR) 13.335], hemoglobin <12.7 g/dl (OR 12.175) and serum total cholesterol <144 mg/dl (OR 8.096) were significant predictors of early VAD requirement. We constructed a scoring system according to the ORs, and the area under the receiver-operating characteristic curve was 0.913 .
\end{abstract}

Conclusions: Low BP, low serum cholesterol and anemia on admission predict early VAD in advanced HF patients who have been treated in non-VAD institutes. Such patients should be promptly referred to a VAD implant center. (Circ J 2014; 78: 2882-2889)

Key Words: INTERMACS profile; Seattle Heart Failure Model; Stage D heart failure

V entricular assist device (VAD) implantation is an important therapeutic option for advanced heart failure (HF) patients. In the REMATCH trial, the use of VAD significantly improved survival in patients with advanced HF with left ventricular ejection fraction (LVEF) $<25 \% .{ }^{1}$ Furthermore, continuous-flow left VAD (LVAD) is proven to accompany better prognosis and quality of life after implantation. ${ }^{2}$

In Japan, implantable LVADs have been approved as bridging devices to transplant since April 2011, and consequently the therapeutic strategy for advanced HF has dramatically changed. ${ }^{3}$ However, in the clinical setting the timing of VAD implantation is always a matter of debate, especially when a patient is referred from a non-VAD institute. Indeed, some referred patients considered to be candidates for VAD implantation eventually recover with intensive medical therapy in VAD centers. In contrast, others need emergency implantation of paracorporeal VAD because their hemodynamic status has crashed before sufficient evaluation for transplant candidacy.

For the purpose of deciding this timing, profiles defined by the Interagency Registry for Mechanically Assisted Circulatory Support (INTERMACS) are useful. Because the prognosis after VAD implantation is worse for patients with profile 1 or 2 than for less sick patients, profile 3 without end-organ dysfunction is the best candidate for VAD implantation. ${ }^{3,4}$ However, the

Received July 10, 2014; revised manuscript received September 12, 2014; accepted September 23, 2014; released online October 30 , 2014 Time for primary review: 60 days

Department of Cardiovascular Medicine (T.F., M.H., H. Muraoka, S.M., T. Inaba, H. Maki, I.K.), Department of Therapeutic Strategy for Heart Failure (K.K., T. Imamura), Department of Cardiothoracic Surgery (O.K., K.N., M.O.), Graduate School of Medicine, University of Tokyo, Tokyo; Division for Health Service Promotion, University of Tokyo, Tokyo (A.Y.), Japan

Mailing address: Koichiro Kinugawa, MD, PhD, Department of Therapeutic Strategy for Heart Failure, Graduate School of Medicine, University of Tokyo, 7-3-1 Hongo, Bunkyo-ku, Tokyo 113-8655, Japan. E-mail: kinugawa-tky@umin.ac.jp

ISSN-1346-9843 doi:10.1253/circj.CJ-14-0749

All rights are reserved to the Japanese Circulation Society. For permissions, please e-mail: cj@j-circ.or.jp 


\begin{tabular}{|c|c|c|c|c|}
\hline & $\begin{array}{l}\text { Total } \\
(n=46)\end{array}$ & $\begin{array}{l}\text { Late/no-VAD } \\
\text { group }(n=20)\end{array}$ & $\begin{array}{c}\text { Early-VAD } \\
\text { group }(n=26)\end{array}$ & $P$ value \\
\hline \multicolumn{5}{|l|}{ Baseline characteristics } \\
\hline Age, years & $39.8 \pm 13.4$ & $43.7 \pm 13.0$ & $36.8 \pm 12.5$ & 0.084 \\
\hline Male, n (\%) & $40(87.0)$ & $18(90.0)$ & $22(84.6)$ & 0.468 \\
\hline Body height, $\mathrm{cm}$ & $168.0 \pm 6.9$ & $167.8 \pm 6.8$ & $168.2 \pm 7.1$ & 0.867 \\
\hline Body weight, kg & $62.0 \pm 11.2$ & $61.1 \pm 9.6$ & $62.8 \pm 12.5$ & 0.613 \\
\hline Body mass index & $22.0 \pm 4.0$ & $21.6 \pm 2.7$ & $22.3 \pm 4.8$ & 0.584 \\
\hline Body surface area, $\mathrm{m}^{2}$ & $1.70 \pm 0.15$ & $1.69 \pm 0.15$ & $1.71 \pm 0.16$ & 0.700 \\
\hline Ischemic etiology $n(\%)$ & $4(8.7)$ & $2(10.0)$ & $2(7.7)$ & 0.590 \\
\hline History of HF, years & $3.3 \pm 4.4$ & $2.6 \pm 3.8$ & $3.8 \pm 4.8$ & 0.361 \\
\hline Family history of HF, $n(\%)$ & $9(19.6)$ & $3(15.0)$ & $6(23.1)$ & 0.383 \\
\hline CRT/ICD, n (\%) & $17(37.0)$ & $4(20.0)$ & $13(50.0)$ & 0.036 \\
\hline$\beta$-blocker, ${ }^{*} \mathrm{mg} /$ day & $6.0 \pm 5.8$ & $5.6 \pm 5.8$ & $6.3 \pm 6.0$ & 0.677 \\
\hline ACEl/ARB, ${ }^{* *} \mathrm{mg} /$ day & $4.3 \pm 4.1$ & $2.5 \pm 2.6$ & $5.7 \pm 4.5$ & 0.006 \\
\hline Statin, n (\%) & $9(19.6)$ & $6(30.0)$ & $3(11.5)$ & 0.117 \\
\hline LVEF <30\%, n (\%) & $42(91.3)$ & $17(85.0)$ & $25(96.2)$ & 0.211 \\
\hline BNP > $300 \mathrm{pg} / \mathrm{ml}, \mathrm{n}(\%)$ & $42(91.3)$ & $17(85.0)$ & $25(96.2)$ & 0.211 \\
\hline INTERMACS profile & - & - & - & $<0.001$ \\
\hline Profile 2, n (\%) & $6(13.0)$ & $0(0)$ & $6(23.1)$ & - \\
\hline Profile 3, n (\%) & $23(50.0)$ & $6(30.0)$ & $17(65.4)$ & - \\
\hline Profile 4 or less sick, $n(\%)$ & $17(37.0)$ & $14(70.0)$ & $3(11.5)$ & - \\
\hline \multicolumn{5}{|c|}{ Clinical course after admission } \\
\hline Paracorporeal VAD, n (\%) & 7 (15.2) & 0 & 7 (26.9) & - \\
\hline Implantable VAD, n (\%) & $21(45.7)$ & $3(15.0)$ & $18(69.2)$ & - \\
\hline All-cause death, n (\%) & $4(8.7)$ & 0 & $4(15.4)$ & - \\
\hline
\end{tabular}

${ }^{*}$ Carvedilol equivalent; **enalapril equivalent.

ACEI, angiotensin-converting enzyme inhibitor; ARB, angiotensin II receptor blocker; BNP, B-type natriuretic peptide; CRT, cardiac resynchronization therapy; HF, heart failure; ICD, implantable cardiac defibrillator; INTERMACS, Interagency Registry for Mechanically Assisted Circulatory Support; LVEF, left ventricular ejection fraction; VAD, ventricular assist device.

classification can vary according to the physician's decision, so we consider that objective parameters that can predict the early requirement for $\mathrm{VAD}$ in advanced $\mathrm{HF}$ patients are essential.

In this study, we focused on objective parameters of advanced $\mathrm{HF}$ patients at the time of referral from non-VAD institutes by analyzing the factors related to early necessity for VAD implantation.

\section{Methods}

\section{Patient Selection}

We retrospectively collected the data for 46 consecutive HF patients who had been referred to the University of Tokyo Hospital from non-VAD institutes for further management of HF between January 2011 and December 2013. The study period was set as described because implantable LVADs were approved in April 2011. The inclusion criteria were defined as follows: $<65$ years old, and history of hospitalization for $\mathrm{HF}$ in a non-VAD institute within 1 month before referral. Patients who were older than 65 years old were excluded because they are ineligible for heart transplantation in Japan and we excluded patients transferred with mechanical circulatory support because they usually need paracorporeal VAD immediately after transfer and thus were not primarily considered as good candidates for implantable LVAD. Patients diagnosed with acute myocarditis, transferred for the purpose of valvular or bypass surgery, or considered ineligible for VAD implantation (ie, social problems) were also excluded.

\section{Variables Evaluated}

Patients' demographic data, including vital signs and treatment on admission, were collected. To evaluate the effects of differential doses of $\beta$-blockers, angiotensin-converting enzyme inhibitors (ACEIs), and angiotensin II receptor blockers (ARBs), the doses were normalized to approximately equivalent doses of carvedilol and enalapril according to efficacy. We regarded bisoprolol $5 \mathrm{mg}$ as carvedilol $20 \mathrm{mg}$, and for ACEIs/ARB, the following agents were regarded as enalapril $10 \mathrm{mg}$ : imidapril $10 \mathrm{mg}$, perindopril $4 \mathrm{mg}$, losartan $50 \mathrm{mg}$, valsartan $80 \mathrm{mg}$, as previously described. ${ }^{5}$ All laboratory data and echocardiographic parameters were obtained within several days of admission. We defined VAD implantation or death as the primary endpoint.

\section{Follow-up After Referral}

Before deciding to implant a VAD, all patients received guideline-directed medical therapy consisting of $\beta$-blockers, ACEIs/ $\mathrm{ARB}$, and aldosterone antagonists as tolerated. Diuretics were also titrated as needed. Cardiac resynchronization therapy (CRT) with a defibrillator was performed if indicated. Intra-aortic balloon pumping (IABP), percutaneous cardiopulmonary support, continuous hemodiafiltration, or mechanical ventilation was executed on the basis of the physician's decision. Timing of VAD implantation was determined by whether the patient developed cardiogenic shock, had progressive decline of end-organ function in spite of maximal treatment, or was considered inotropedependent despite optimal medical therapy.

We followed all patients from the date of their referral until 

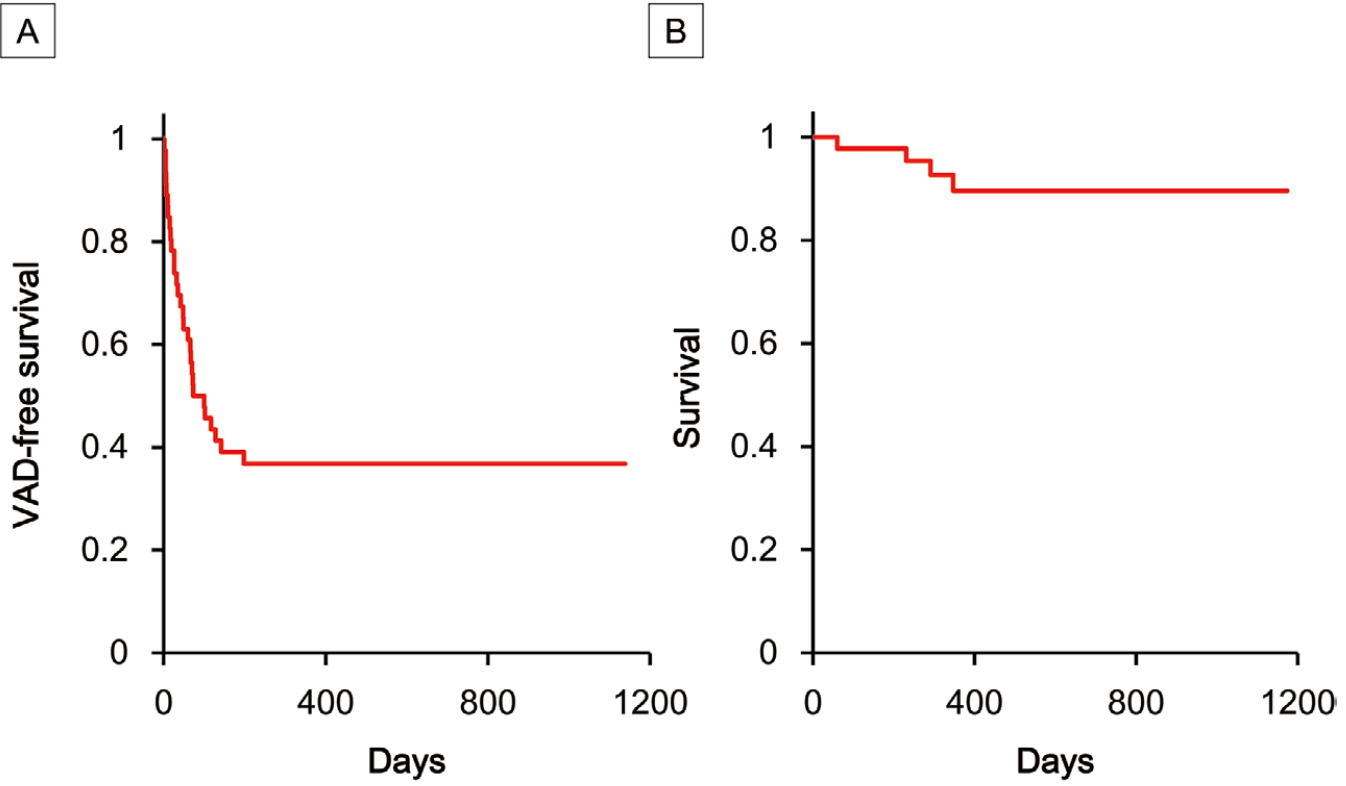

Figure 1. Kaplan-Meier curve for VAD-free survival (A) and survival (B) after admission to referral hospital of all enrolled patients $(n=46)$ with advanced heart failure. VAD, ventricular assist device.

March 2014 or the date of their death. No patient was lost during the follow-up period unless deceased.

\section{Previous Risk Scores}

The Seattle Heart Failure Model (SHFM) was developed by Levy et al from the data of New York Heart Association (NYHA) class IIIB or IV patients and calculated from age, vital signs, medications, laboratory measurements and utilization of CRT. ${ }^{6}$ They also updated the SHFM score for LVAD candidates by adding variables such as usage of inotropes, IABP, or mechanical ventilation. ${ }^{7}$ In SHFM, the therapeutic option of LVAD is available if the patients meet ACC/AHA criteria such as (1) NYHA Class IV, (2) LVEF $\leq 25 \%$, and (3) mean 2 year survival $\leq 50 \% .{ }^{8}$ We used their website (http://depts. washington.edu/shfm) to estimate the indication for LVAD in the present group of patients.

The INTERMACS profile stratifies patients with advanced HF into 7 categories on the basis of clinical severity. ${ }^{9}$ In this study, patients with profile 1 on admission were not enrolled because we excluded patients transferred with mechanical circulatory support.

\section{Statistical Analysis}

Continuous variables of patients who did or did not require early VAD were compared by unpaired t-test or Mann-Whitney test as appropriate. Categorical variables of patients were compared by chi-square test or Fisher's exact test as appropriate. Univariable analyses with a logistic regression model were performed to calculate odds ratio (OR) and $95 \%$ confidence interval $(\mathrm{CI})$ to assess the influence of each variable on admission on early requirement for $\mathrm{VAD}$. We chose 3 statistically significant variables from among the baseline characteristics, laboratory measurements and echocardiographic parameters. Cutoff points of the 3 variables were determined by receiver-operating characteristic (ROC) analysis with JMP9 (SAS Institute, Cary,
NC, USA) and entered into the multivariable analysis.

Considering the results of the multivariable analysis, weighted scores were allocated to each selected variable on the basis of each OR, and we defined a summation of scores as a new risk scoring system. We then created a ROC curve for the new score, by which the area under the curve (AUC) for the score was calculated and that AUC was compared with those of previously published risk scores, including SHFM and INTERMACS profile.

Kaplan-Meier survival analysis was also performed to evaluate VAD-free survival after referral on 2 strata of the new score, and VAD-free survival among these strata was compared by log-rank test.

Unless otherwise specified, all data are expressed as mean \pm standard deviation. Probability was 2-tailed, with $\mathrm{P}<0.05$ regarded as statistically significant. All statistical analyses except for the ROC analyses were calculated with PASW Statistics 18 (SPSS, Chicago, IL, USA).

\section{Results}

\section{Baseline Characteristics}

The baseline characteristics of the 46 enrolled patients are shown in Table 1. The mean age was $39.8 \pm 13.4$ years and $87.0 \%$ were male. An ischemic etiology was found in $8.7 \%$ of the patients; 6 patients $(13.0 \%)$ were considered as profile 2 and $23(50.0 \%)$ as profile 3 . LVEF was $<30 \%$ in 42 patients $(91.3 \%)$, and plasma levels of B-type natriuretic peptide (BNP) were $>300 \mathrm{pg} / \mathrm{ml}$ in 42 patients $(91.3 \%)$.

\section{Classification of the Patients}

Table 1 shows the clinical course after admission, and Figure 1 shows the VAD-free survival and overall survival of the patients. As shown in Figure 1A, 28 patients $(60.9 \%)$ eventually needed VAD and of them 25 had the VAD implanted within 4 months 
Table 2. Demographic, Physical, Laboratory and Echocardiographic Parameters on Admission and Univariable Analysis for VAD Implantation in 46 Patients With Advanced HF

\begin{tabular}{|c|c|c|c|c|c|}
\hline & $\begin{array}{l}\text { Late/no-VAD } \\
\text { group }(n=20)\end{array}$ & $\begin{array}{c}\text { Early-VAD } \\
\text { group }(n=26)\end{array}$ & $P$ value & OR & $95 \% \mathrm{Cl}$ \\
\hline \multicolumn{6}{|l|}{ Demographic parameters } \\
\hline Age, years & $43.7 \pm 13.0$ & $36.8 \pm 12.5$ & 0.089 & 0.959 & $0.914-1.006$ \\
\hline Male, $\mathrm{n}(\%)$ & $18(90.0)$ & $22(84.6)$ & 0.593 & 1.636 & $0.268-9.980$ \\
\hline Body height, $\mathrm{cm}$ & $167.8 \pm 6.8$ & $168.2 \pm 7.1$ & 0.863 & 1.008 & $0.925-1.097$ \\
\hline Body weight, kg & $61.1 \pm 9.6$ & $62.8 \pm 12.5$ & 0.605 & 1.014 & $0.962-1.069$ \\
\hline Body mass index & $21.6 \pm 2.7$ & $22.3 \pm 4.8$ & 0.577 & 1.044 & $0.897-1.216$ \\
\hline Body surface area, $\mathrm{m}^{2}$ & $1.69 \pm 0.15$ & $1.71 \pm 0.16$ & 0.693 & 2.166 & $0.047-100.583$ \\
\hline Ischemic etiology, $\mathrm{n}(\%)$ & $2(10.0)$ & $2(7.7)$ & 0.784 & 0.750 & $0.096-5.844$ \\
\hline History of HF, years & $2.6 \pm 3.8$ & $3.8 \pm 4.8$ & 0.357 & 1.070 & $0.926-1.237$ \\
\hline Family history of HF, n (\%) & $3(15.0)$ & $6(23.1)$ & 0.496 & 1.700 & $0.368-7.845$ \\
\hline \multicolumn{6}{|l|}{ Physical parameters } \\
\hline Heart rate, beats/min & $88.8 \pm 18.0$ & $93.4 \pm 19.8$ & 0.413 & 1.013 & $0.982-1.046$ \\
\hline Systolic BP, mmHg & $95.9 \pm 12.8$ & $88.0 \pm 7.8$ & 0.024 & 0.924 & $0.862-0.989$ \\
\hline Systolic BP <93 mmHg, n (\%) & 7 (35.0) & $23(88.5)$ & 0.001 & 14.238 & $3.133-64.702$ \\
\hline Diastolic BP, mmHg & $60.2 \pm 8.8$ & $54.6 \pm 7.8$ & 0.040 & 0.916 & $0.842-0.996$ \\
\hline \multicolumn{6}{|l|}{ Laboratory parameters } \\
\hline White blood cell count, $\times 10^{3} / \mu \mathrm{l}$ & $7.4 \pm 2.5$ & $7.2 \pm 2.2$ & 0.708 & 0.953 & $0.741-1.226$ \\
\hline Hemoglobin, g/dl & $14.6 \pm 2.0$ & $12.4 \pm 2.1$ & 0.002 & 0.585 & $0.413-0.828$ \\
\hline Hemoglobin $<12.7 \mathrm{~g} / \mathrm{dl}, \mathrm{n}(\%)$ & $2(10.0)$ & $17(65.4)$ & 0.001 & 17.000 & $3.202-90.254$ \\
\hline Platelets, $\times 10^{4} / \mu \mathrm{l}$ & $21.2 \pm 5.6$ & $22.4 \pm 6.8$ & 0.514 & 1.033 & $0.937-1.138$ \\
\hline Serum sodium, mmol/L & $137.1 \pm 3.9$ & $135.3 \pm 5.4$ & 0.219 & 0.920 & $0.805-1.051$ \\
\hline Serum potassium, $\mathrm{mmol} / \mathrm{L}$ & $4.4 \pm 0.4$ & $4.3 \pm 0.5$ & 0.542 & 0.670 & $0.185-2.429$ \\
\hline Serum chloride, $\mathrm{mmol} / \mathrm{L}$ & $101.6 \pm 4.0$ & $99.3 \pm 6.0$ & 0.156 & 0.910 & $0.798-1.037$ \\
\hline Serum blood urea nitrogen, $\mathrm{mg} / \mathrm{dl}$ & $21.0 \pm 11.3$ & $23.3 \pm 14.6$ & 0.548 & 1.014 & $0.968-1.063$ \\
\hline Serum creatinine, $\mathrm{mg} / \mathrm{dl}$ & $1.00 \pm 0.25$ & $1.12 \pm 0.51$ & 0.327 & 2.150 & $0.465-9.938$ \\
\hline Serum uric acid, mg/dl & $7.0 \pm 2.2$ & $7.5 \pm 3.2$ & 0.525 & 1.075 & $0.861-1.341$ \\
\hline Serum total protein, $\mathrm{g} / \mathrm{dl}$ & $7.0 \pm 0.8$ & $6.7 \pm 0.8$ & 0.241 & 0.634 & $0.296-1.359$ \\
\hline Serum albumin, g/dl & $3.9 \pm 0.5$ & $3.7 \pm 0.6$ & 0.371 & 0.608 & $0.204-1.809$ \\
\hline Serum lactate dehydrogenase, IU/L & $245.3 \pm 58.5$ & $276.4 \pm 113.5$ & 0.287 & 1.004 & $0.996-1.013$ \\
\hline Serum aspartate aminotransferase, IU/L & $32.2 \pm 13.1$ & $36.1 \pm 38.8$ & 0.666 & 1.005 & $0.983-1.027$ \\
\hline Serum alanine aminotransferase, IU/L & $36.2 \pm 20.2$ & $41.4 \pm 52.5$ & 0.670 & 1.003 & $0.988-1.019$ \\
\hline Serum alkaline phosphatase, IU/L & $278.1 \pm 75.8$ & $288.5 \pm 111.9$ & 0.724 & 1.001 & $0.995-1.008$ \\
\hline Serum $\gamma$-glutamyl transpeptidase, IU/L & $117.2 \pm 104.7$ & $107.3 \pm 88.3$ & 0.724 & 0.999 & $0.993-1.005$ \\
\hline Serum total bilirubin, $\mathrm{mg} / \mathrm{dl}$ & $1.4 \pm 0.7$ & $1.5 \pm 0.9$ & 0.663 & 1.174 & $0.570-2.418$ \\
\hline Serum TC, mg/dl & $181.0 \pm 43.8$ & $146.2 \pm 46.3$ & 0.020 & 0.983 & $0.969-0.997$ \\
\hline Serum TC $<144 \mathrm{mg} / \mathrm{dl}, \mathrm{n}(\%)$ & $3(15.0)$ & $16(61.5)$ & 0.003 & 9.067 & $2.106-39.029$ \\
\hline C-reactive protein, $\mathrm{mg} / \mathrm{dl}$ & $0.78 \pm 1.30$ & $1.42 \pm 2.34$ & 0.289 & 1.223 & $0.843-1.774$ \\
\hline BNP, $\log _{10} \mathrm{pg} / \mathrm{ml}$ & $2.84 \pm 0.34$ & $2.96 \pm 0.25$ & 0.201 & 3.960 & $0.480-32.676$ \\
\hline \multicolumn{6}{|l|}{ Echocardiographic parameters } \\
\hline LV diastolic diameter, mm & $67.5 \pm 16.2$ & $73.1 \pm 14.3$ & 0.228 & 1.027 & $0.984-1.072$ \\
\hline LV systolic diameter, mm & $63.1 \pm 11.6$ & $67.5 \pm 14.5$ & 0.263 & 1.027 & $0.980-1.077$ \\
\hline LVEF, \% & $21.7 \pm 10.5$ & $20.2 \pm 6.9$ & 0.545 & 0.979 & $0.913-1.049$ \\
\hline Interventricular septum thickness, $\mathrm{mm}$ & $8.3 \pm 2.3$ & $7.8 \pm 1.8$ & 0.416 & 0.884 & $0.657-1.189$ \\
\hline Posterior wall thickness, $\mathrm{mm}$ & $7.9 \pm 2.0$ & $8.2 \pm 1.3$ & 0.546 & 1.120 & $0.775-1.617$ \\
\hline LA diameter, $\mathrm{mm}$ & $50.1 \pm 6.7$ & $48.0 \pm 7.3$ & 0.341 & 0.959 & $0.879-1.045$ \\
\hline AR severity & $0.50 \pm 0.76$ & $0.58 \pm 0.70$ & 0.717 & 1.165 & $0.510-2.659$ \\
\hline MR severity & $2.55 \pm 0.69$ & $2.31 \pm 1.01$ & 0.355 & 0.725 & $0.367-1.433$ \\
\hline TR severity & $1.95 \pm 0.69$ & $2.00 \pm 0.85$ & 0.826 & 1.089 & $0.507-2.340$ \\
\hline TR pressure gradient, $\mathrm{mmHg}$ & $30.3 \pm 9.5$ & $37.2 \pm 14.6$ & 0.086 & 1.048 & $0.993-1.106$ \\
\hline
\end{tabular}

$\mathrm{AR}$, aortic regurgitation; $\mathrm{BP}$, blood pressure; $\mathrm{Cl}$, confidence interval; $\mathrm{LA}$, left atrial; $\mathrm{LV}$, left ventricular; $\mathrm{MR}$, mitral regurgitation; OR, odds ratio; TC, total cholesterol; TR, tricuspid regurgitation. Other abbreviations as in Table 1. 


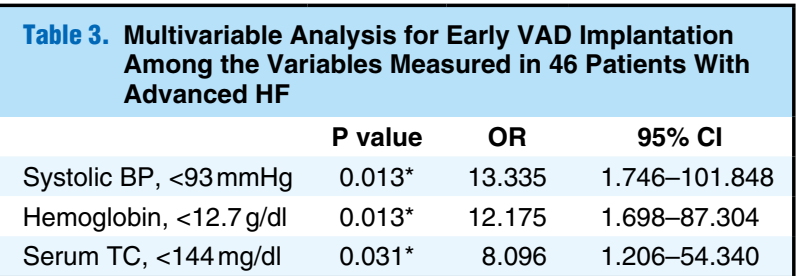

Abbreviations as in Tables 1,2.

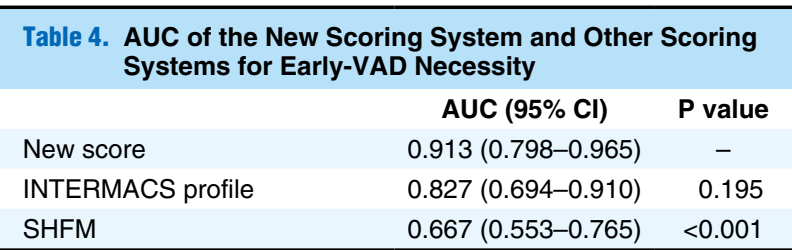

AUC, area under the curve;SHFM, Seattle Heart Failure Model. Other abbreviations as in Tables 1,2.

of admission; 1 patient died within the 4 months, before VAD implantation. Next, we classified the patients who had a VAD implanted or died before day 120 into an early-VAD group $(n=26)$, and a late/no-VAD group $(n=20)$. Status on admission and the clinical course of both groups are also shown in Table 1. Patients in the early-VAD group tended to be younger than those in the late/no-VAD group, though statistically insignificant ( $36.8 \pm 12.5$ vs. $43.7 \pm 13.0$ years, $\mathrm{P}=0.084)$. The initial dose of $\beta$-blockers on admission was comparable between groups $(6.3 \pm 6.0 \mathrm{mg} /$ day in early-VAD group vs. $5.6 \pm 5.8 \mathrm{mg} / \mathrm{day}$ in late/ no-VAD group, $\mathrm{P}=0.677$ ) but the doses of $\mathrm{ACEIs/ARB}$ were significantly higher in the early-VAD group $(5.7 \pm 4.5 \mathrm{mg} /$ day vs $2.5 \pm 2.6 \mathrm{mg} / \mathrm{day}, \mathrm{P}=0.006)$. $\mathrm{CRT} / \mathrm{implantable} \mathrm{cardiac} \mathrm{defi-}$ brillator implantation was performed in 13 patients $(50.0 \%)$ in the early-VAD group and in 4 patients $(20.0 \%)$ from the late/ no-VAD group $(\mathrm{P}=0.036)$.

In the early-VAD group, a paracorporeal VAD was implanted in 7 patients as an emergency life-saving procedure in all cases, and an implantable VAD in 18 patients. One patient died before VAD implantation. In the late/no-VAD group, 3 patient had a VAD implanted after day 120. In the follow-up period until March 2014, 3 patients in the early-VAD group died after VAD implantation (all of them had received an implantable VAD), whereas no patients died in the late/no-VAD group.

\section{Univariable Logistic Analysis}

We performed univariable logistic analysis to find predictors for early VAD. Table 2 shows the demographic and physical parameters on admission. Low systolic blood pressure (BP; $\mathrm{P}=0.024$, OR $0.924,95 \%$ CI $0.862-0.989$ ) was a significant factor related to the necessity for VAD. Of the laboratory data (Table 2), low hemoglobin level ( $\mathrm{P}=0.002$, OR 0.585 , 95\% CI $0.413-0.828)$ and low serum total cholesterol level $(\mathrm{P}=0.020$, OR $0.983,95 \%$ CI $0.969-0.997)$ predicted the early necessity for VAD. Serum bilirubin and creatinine levels, which are the well-known markers of end-organ dysfunction, or the plasma BNP level on admission were not significantly related to early VAD necessity. Of the echocardiographic parameters (Table 2), none of the variables, including left ventricular diastolic diameter and LVEF, were significantly related to early VAD necessity.

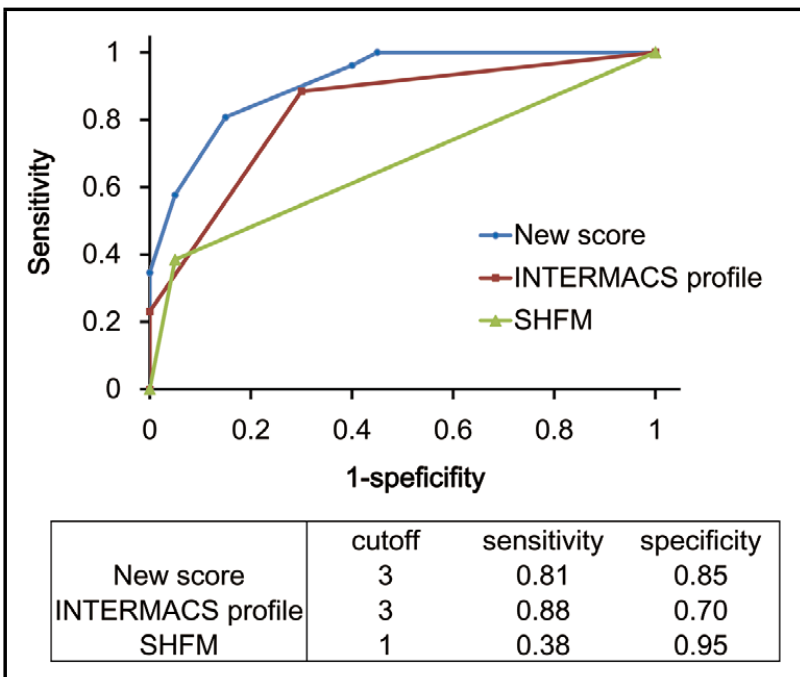

Figure 2. ROC analysis of the new score, the INTERMACS profile and SHFM for the prediction of VAD necessity within 120 days, with the sensitivity and specificity of each score at the cutoff point. ROC, receiver-operating characteristics; INTERMACS, Interagency Registry for Mechanically Assisted Circulatory Support; SHFM, Seattle Heart Failure Model; VAD, ventricular assist device.

group, we chose all 3 statistically significant variables for the multivariable analysis. Systolic $\mathrm{BP}<93 \mathrm{mmHg}(\mathrm{P}=0.001$, OR $14.238,95 \%$ CI 3.133-64.702), hemoglobin $<12.7 \mathrm{~g} / \mathrm{dl}$ ( $\mathrm{P}=0.001$, OR 17.000, 95\% CI 3.202-90.254) and serum total cholesterol $<144 \mathrm{mg} / \mathrm{dl}$ ( $\mathrm{P}=0.003$, OR 9.067, 95\% CI 2.106-39.029) were selected (Table 2) and entered into the multivariable analysis, which found that all of them were independent predictors for early VAD necessity (Table 3 ).

\section{Scoring System}

We created a new scoring system using these 3 factors (Table 3 ). We assigned 2 points to systolic $\mathrm{BP}<93 \mathrm{mmHg}, 2$ points to hemoglobin $<12.7 \mathrm{~g} / \mathrm{dl}$ and 1 point to serum total cholesterol $<144 \mathrm{mg} / \mathrm{dl}$, based on the ORs in the multivariable analysis. A ROC curve of the score was drawn, and the AUC for the score was 0.913 (Figure 2, Table 4). With a cutoff of 3 points, the sensitivity for VAD necessity within 120 days was 0.81 and the specificity was 0.85 . We also compared the predictability of this score with other risk scores. The AUC of INTERMACS profile was 0.827 , and with a cutoff of profile 3 the sensitivity was 0.88 and the specificity was 0.70 . The AUC of SHFM was 0.667 , and the sensitivity and specificity were 0.38 and 0.95 , respectively. Our new scoring had a significantly better predictability for the early necessity of VAD over SHFM (Table 4).

We also classified the patients into 2 strata according to the new score (score 0-2 or 3-5), and performed Kaplan-Meier analysis. Figure $3 \mathrm{~A}$ shows that the VAD-free survival was significantly different between the 2 strata $(\mathrm{P}<0.001$, by log-rank test). We also compared VAD-free survival after stratification with the INTERMACS profile and SHFM (Figures 3B,C). VAD-free survival after admission was also significantly different between the 2 strata according to INTERMACS profile (profile 2-3 and profile 4 or less sick, $\mathrm{P}<0.001$ ) and $\mathrm{SHFM}$ (LVAD criteria (+) and (-), $\mathrm{P}<0.001)$.

\section{Multivariable Logistic Analysis}

Because 26 primary events were observed in the early-VAD 


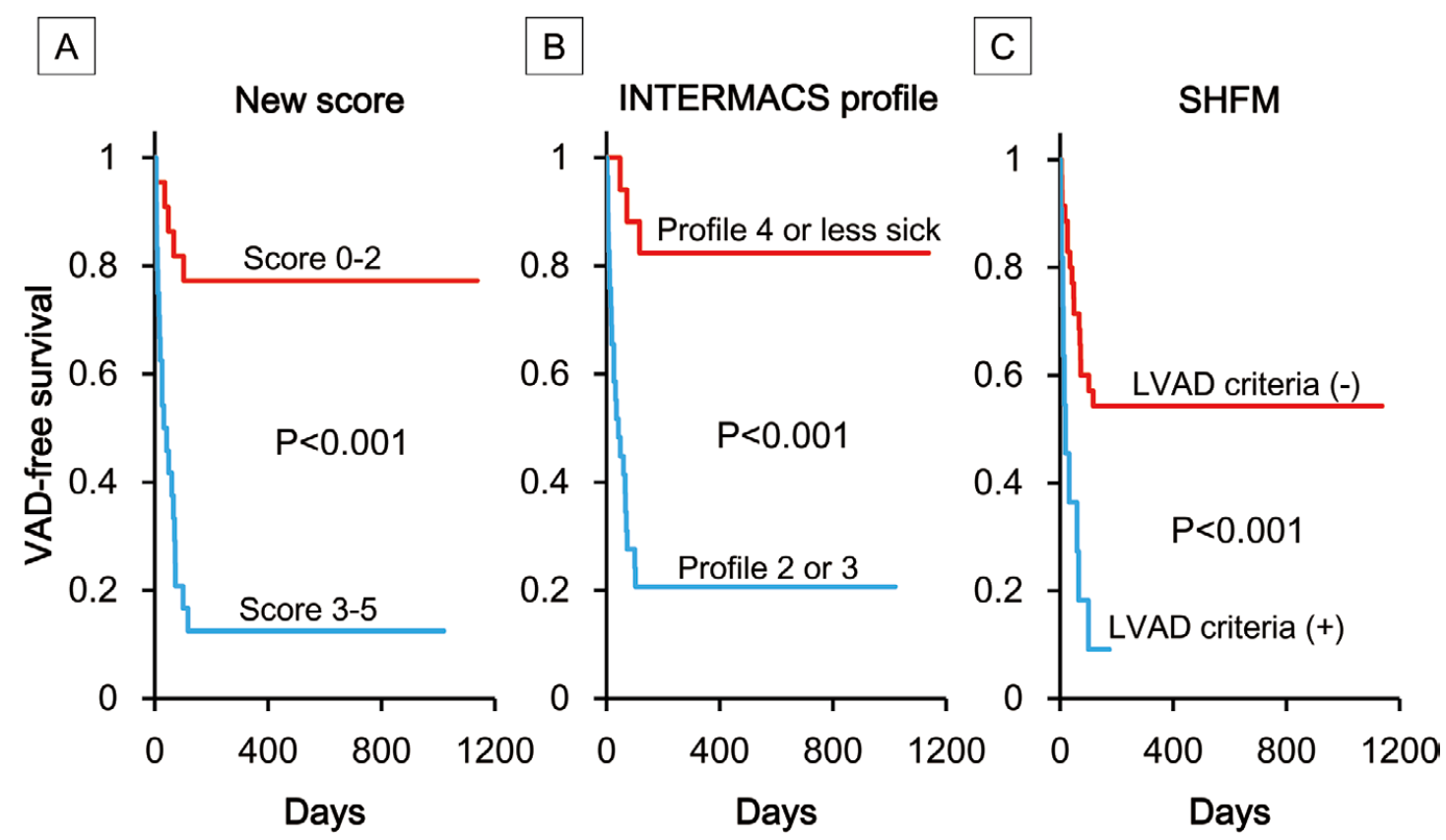

Figure 3. Kaplan-Meier analysis of VAD-free survival according to the new score system (A), profile 2 or 3 and profile 4 or less sick in INTERMACS $(\mathbf{B})$, and LVAD criteria $(-)$ and $(+)$ in SHFM (C). VAD-free survival was compared by log-rank test and each $P$ value is shown. INTERMACS, Interagency Registry for Mechanically Assisted Circulatory Support; LVAD, left ventricular assist device; SHFM, Seattle Heart Failure Model.

\section{Discussion}

This study demonstrated that low systolic BP, and low levels of hemoglobin and low serum total cholesterol on admission were independent predictors of subsequent VAD implantation in the not too distant future for advanced HF patients referred from non-VAD institutes. For the purpose of deciding the timing of VAD implantation, it has been suggested that profiles defined by INTERMACS are useful, ${ }^{3,4}$ but the decision is physiciandependent. We emphasize that the combination of 3 objectively defined parameters was similarly predictive to INTERMACS profile. There have been several risk scores proposed for predicitng prognosis after VAD implantation, ${ }^{7,10-12}$ but to our knowledge, this is the first report to focus on the early necessity for VAD using objective parameters. We also underscore that we selected noninvasive variables. Hemodynamic parameters obtained invasively may be useful for determining the timing, but are often unavailable at the time of referral from non-VAD institutes.

In this report, we use the term "at the time of referral". In reality, we collected the data at the time of admission to the referral hospital, and so to be exact, we use the term "at the time of admission to the referral hospital". However, we consider it was easier to understand the meaning and importance of this report by using the term "at the time of referral".

Clinical Course of Advanced HF Patients Referred to Hospital According to our results, VAD-free survival was only $43.5 \%$ at 4 month after admission (Figure 1). Furthermore, the existence of 2 of the 3 factors (systolic BP $<93 \mathrm{mmHg}$, hemoglobin $<12.7 \mathrm{~g} / \mathrm{dl}$ and serum total cholesterol $<144 \mathrm{mg} / \mathrm{dl}$ ) predicted early VAD implantation after admission (Figure 2). Kaplan-
Meier analysis showed these patients had extremely poor VADfree survival (Figure 3), which was comparable with the VADfree survival of status 1 patients after being listed for heart transplantation as we reported recently. ${ }^{13}$

\section{Effect of Each Parameter on Patients' Outcomes}

It has been previously shown that low $\mathrm{BP},{ }^{14,15}$ anemia ${ }^{16,17}$ and low serum total cholesterol ${ }^{18,19}$ are predictors of higher mortality in HF patients, which is consistent with our results, because patients who needed VAD were expected to have poor prognosis without VAD implantation.

Patients presenting with low systolic BP may have low cardiac output and impaired organ perfusion, ${ }^{20}$ so it is reasonable that low systolic BP predicts HF severity and the patient's outcome. In this study, the dose of ACEIs/ARB in early-VAD patients was higher than that in late/non-VAD patients (Table 1), which could be related to the duration of HF treatment, and could be a cause of the lower BP in early-VAD patients.

$\mathrm{BP}$ is an objective, but fluctuating parameter. We used BP measured noninvasively after admission with the patient in a stable condition and supine position. Although the difference in systolic BP between early-VAD and late/no-VAD patients was small, we consider the cutoff value of $93 \mathrm{mmHg}$ as clinically reasonable, considering that systolic $\mathrm{BP}<90 \mathrm{mmHg}$ is commonly regarded as a state of shock.

Low serum total cholesterol is a novel predictor of survival in chronic HF, but the mechanism is not fully understood. ${ }^{18} \mathrm{In}$ our study the dose of statin was not statistically different, but tended to be higher in the patients in the late/no-VAD group than in those in early-VAD group, although the total cholesterol level was significantly lower in the early-VAD group (Tables 1,2). The protective role of lipoproteins in downregu- 
lating inflammatory cytokines in HF has been reported. ${ }^{21}$ On the other hand, it is possible that low cholesterol is a marker only for cachexia, a state associated with poor HF mortality, and has no pathophysiologic role. However, in our study low cholesterol was a predictor of early VAD necessity, independent of body mass index or serum albumin, which are also variables associated with poor nutritional condition (data not shown). Therefore, cardiac cachexia may not only be the cause of low serum total cholesterol in our study.

Anemia is common in patients with HF, and patients with both HF and anemia have a lower functional capacity, worse quality of life, and higher rates of hospitalization and death than those without anemia. ${ }^{22,23}$ There are several potential causes of anemia in HF: malnutrition; hemodilution; renal dysfunction and impaired erythropoietin production; usage of ACEIs/ARB; elevated levels of proinflammatory cytokines; and others. ${ }^{24} \mathrm{We}$ found that anemia in the early-VAD group was normocytic and none had undergone blood transfusion prior to referral (data not shown). Renal function was comparable between the earlyVAD and late/no-VAD groups (Table 2). We did not have enough data about iron metabolism. Right atrial pressure and pulmonary capillary wedge pressure by cardiac catheterization were significantly higher in the early-VAD group (Table S1). We speculate that hemodilution because of systemic congestion at least partially caused the anemia in the early-VAD group.

\section{Pitfalls of Previous Risk Scores}

This study revealed that the INTERMACS profile 3 or greater on admission was another good predictor of early VAD necessity, and the results were consistent with previous recommendations for VAD implantation. ${ }^{3,4}$ However, stratification by these profiles is largely dependent on the physician's decision. In fact, we often suggest that physicians in non-VAD institutes start inotropes before transfer of their patients in order to avoid end-organ dysfunction. Two patients in our study became 'profile 3' just before transfer because of this suggestion. We consider that a scoring system composed of factors that are independent of the decision or intervention by physicians is preferable. In this regard, the scoring system that we newly created is valuable because we selected objective parameters that can be obtained noninvasively.

\section{Is the Current Referral Timing Appropriate?}

In Japan, implantable LVADs are available only as a bridge to transplantation, so being listed for heart transplantation is required before implantable LVAD therapy can be performed. Evaluation of eligibility for heart transplant usually takes more than 1 month, and we consider that the current timing of referral is still too late for implantable LVAD therapy. The delay in referral, at least in part, caused the need for life-saving paracorporeal VAD in 7 patients as well as 1 death during evaluation. Although all-cause mortality was not significantly different between the groups stratified by the new scoring system or by INTERMACS profile (data not shown), it is not true that patients are best referred after they have accumulated a high score. We have not yet established the best timing of referral to VAD implant centers, but we would like to emphasize that the referral timing of the patients in early-VAD group was not soon enough for implantable LVAD therapy.

\section{Study Limitations}

We acknowledge that our study has several limitations. First, this study was conducted in a single center, and consequently included a limited number of patients. The timing of the decision for VAD implantation may be different in different insti- tutes. Second, we cannot yet propose appropriate timing of referral for implantable VAD therapy, including preoperative listing for transplant. Because the timing of referral is always a matter of debate, especially for physicians in non-VAD institutes, further investigations such as prospective studies or large-scale surveys are anticipated to establish the best timing of referral. Finally, in this study advanced HF with an ischemic etiology was found in only 4 patients $(8.7 \%)$, which is a remarkable feature specific to the Japanese population requiring VAD for advanced HF. Other factors may be found in other populations.

\section{Conclusions}

Low BP, low serum total cholesterol level and anemia on admission were factors that predicted the early necessity for VAD in advanced HF patients referred from non-VAD institutes. Such patients should be promptly referred for VAD implantation.

\section{Disclosures}

Grants: None. We have no conflict of interest to disclosure.

\section{References}

1. Rose EA, Gelijns AC, Moskowitz AJ, Heitjan DF, Stevenson LW, Dembitsky W, et al. Long-term use of a left ventricular assist device for end-stage heart failure. N Engl J Med 2001; 345: 1435-1443.

2. Slaughter MS, Rogers JG, Milano CA, Russell SD, Conte JV, Feldman $\mathrm{D}$, et al. Advanced heart failure treated with continuous-flow left ventricular assist device. N Engl J Med 2009; 361: 2241-2251.

3. Kinugawa K. How to treat stage D heart failure?: When to implant left ventricular assist devices in the era of continuous flow pumps? Circ J 2011; 75: 2038-2045.

4. Kirklin JK, Naftel DC, Pagani FD, Kormos RL, Stevenson LW, Blume ED, et al. Sixth INTERMACS Annual Report: A 10,000-patient database. J Heart Lung Transplant 2014; 33: 555-564.

5. Kato N, Kinugawa K, Imamura T, Muraoka H, Minatsuki S, Inaba $\mathrm{T}$, et al. Trend of clinical outcome and surrogate markers during titration of $\beta$-blocker in heart failure patients with reduced ejection fraction: Relevance of achieved heart rate and $\beta$-blocker dose. Circ J 2013; 77: 1001-1008.

6. Levy WC, Mozaffarian D, Linker DT, Sutradhar SC, Anker SD, Cropp $\mathrm{AB}$, et al. The Seattle Heart Failure Model: Prediction of survival in heart failure. Circulation 2006; 113: 1424-1433.

7. Levy WC, Mozaffarian D, Linker DT, Farrar DJ, Miller LW. Can the Seattle Heart Failure Model be used to risk-stratify heart failure patients for potential left ventricular assist device therapy? J Heart Lung Transplant 2009; 28: 231-236.

8. Yancy CW, Jessup M, Bozkurt B, Butler J, Casey DE Jr, Drazner $\mathrm{MH}$, et al. 2013 ACCF/AHA guideline for the management of heart failure: A report of the American College of Cardiology Foundation/ American Heart Association Task Force on Practice Guidelines. Circulation 2013; 128: e240-e327, doi:10.1161/CIR.0b013e31829e8776.

9. Kirklin JK, Naftel DC, Stevenson LW, Kormos RL, Pagani FD, Miller MA, et al. INTERMACS database for durable devices for circulatory support: First annual report. J Heart Lung Transplant 2008; 27: $1065-1072$.

10. Rao V, Oz MC, Flannery MA, Catanese KA, Argenziano M, Naka Y. Revised screening scale to predict survival after insertion of a left ventricular assist device. J Thorac Cardiovasc Surg 2003; 125: 855862.

11. Lietz K, Long JW, Kfoury AG, Slaughter MS, Silver MA, Milano $\mathrm{CA}$, et al. Outcomes of left ventricular assist device implantation as destination therapy in the post-rematch era: Implications for patient selection. Circulation 2007; 116: 497-505.

12. Imamura T, Kinugawa K, Shiga T, Endo M, Kato N, Inaba T, et al. Novel risk scoring system with preoperative objective parameters gives a good prediction of 1-year mortality in patients with a left ventricular assist device. Circ J 2012; 76: 1895-1903.

13. Imamura T, Kinugawa K, Hatano M, Fujino T, Inaba T, Maki H, et al. Status 2 patients had poor prognosis without mechanical circulatory support. Circ J 2014; 78: 1396-1404.

14. Gheorghiade M, Abraham WT, Albert NM, Greenberg BH, O'Connor $\mathrm{CM}$, She L, et al. Systolic blood pressure at admission, clinical characteristics, and outcomes in patients hospitalized with acute heart fail- 
ure. JAMA 2006; 296: 2217-2226.

15. Ambrosy AP, Vaduganathan M, Mentz RJ, Greene SJ, Subacius H, Konstam MA, et al. Clinical profile and prognostic value of low systolic blood pressure in patients hospitalized for heart failure with reduced ejection fraction: Insights from the efficacy of vasopressin antagonism in heart failure: Outcome study with tolvaptan (EVEREST) trial. Am Heart J 2013; 165: 216-225.

16. Ezekowitz JA, McAlister FA, Armstrong PW. Anemia is common in heart failure and is associated with poor outcomes: Insights from a cohort of 12065 patients with new-onset heart failure. Circulation 2003; 107: 223-225.

17. Groenveld HF, Januzzi JL, Damman K, van Wijngaarden J, Hillege HL, van Veldhuisen DJ, et al. Anemia and mortality in heart failure patients: A systematic review and meta-analysis. J Am Coll Cardiol 2008; 52: 818-827.

18. Horwich TB, Hamilton MA, Maclellan WR, Fonarow GC. Low serum total cholesterol is associated with marked increase in mortality in advanced heart failure. J Card Fail 2002; 8: 216-224.

19. Afsarmanesh N, Horwich TB, Fonarow GC. Total cholesterol levels and mortality risk in nonischemic systolic heart failure. Am Heart $J$ 2006; 152: 1077-1083.

20. Gheorghiade M, Zannad F, Sopko G, Klein L, Pina IL, Konstam MA, et al. Acute heart failure syndromes: Current state and framework for future research. Circulation 2005; 112: 3958-3968.

21. Rauchhaus M, Coats AJ, Anker SD. The endotoxin-lipoprotein hypothesis. Lancet 2000; 356: 930-933.

22. Swedberg K, Young JB, Anand IS, Cheng S, Desai AS, Diaz R, et al. Treatment of anemia with darbepoetin alfa in systolic heart failure. N Engl J Med 2013; 368: 1210-1219.

23. Maggioni AP, Opasich C, Anand I, Barlera S, Carbonieri E, Gonzini $\mathrm{L}$, et al. Anemia in patients with heart failure: Prevalence and prognostic role in a controlled trial and in clinical practice. J Card Fail 2005; 11: $91-98$.

24. Silverberg DS, Wexler D, Iaina A. The importance of anemia and its correction in the management of severe congestive heart failure. Eur J Heart Fail 2002; 4: 681-686.

\section{Supplementary Files}

Supplementary File 1

Table S1. Hemodynamic parameters of the 46 enrolled patients with advanced heart failure

Please find supplementary file(s);

http://dx.doi.org/10.1253/circj.CJ-14-0749 